\title{
Análisis de la estructura organizacional del consultorio Innovadental a partir de la matriz DOFA
}

\author{
Yarlen Yesid Ibarguen-Mosquera ${ }^{1}$ \\ Corporación Universitaria Minuto de Dios - UNIMINUTO \\ yibarguenm3@uniminuto.edu.co \\ Luz Karime Rueda-Sánchez ${ }^{2}$ \\ Corporación Universitaria Minuto de Dios - UNIMINUTO \\ lruedasanch@uniminto.edu.co \\ Adrian Marcel Garcia-Caicedo ${ }^{3}$ \\ Corporación Universitaria Minuto de Dios - UNIMINUTO \\ adrian.garcia@uniminuto.edu
}

\section{DOI: https://doi.org/10.21158/23227230.v9.n0.2019.2645}

Cómo citar este artículo: Ibarguen-Mosquera, Y. Y.; Rueda-Sánchez, L. K.; Garcia-Caicedo, A. M. (2019). Análisis de la estructura organizacional del consultorio Innovadental a partir de la matriz DOFA. Revista Ploutos, 9, (Páginas). DOI: https://doi.org/10.21158/23227230.v9.n0.2019.2645

Fecha de recepción: 06 de abril de 2019

Fecha de aprobación: 10 de julio de 2020

\section{Resumen}

Este trabajo presenta los resultados del cuadrante de vulnerabilidad del consultorio odontológico Innovadental, luego de realizar un análisis de la estructura organizacional del consultorio a partir de la matriz DOFA. Se utilizaron los cuadrantes del diagrama de vulnerabilidad y el trazo de puntales para determinar el grado en el que se encuentra la organización. Este método es un instrumento de planificación estratégica usado en el estudio con el fin de explorar el entorno, con la identificación de factores internos y externos previstos para la búsqueda de una planificación y la determinación de acciones de mejora que ayuden al crecimiento y al fortalecimiento de la empresa, identificando debilidades, oportunidades, fortalezas y amenazas, así como reconocer el estado actual de la organización. Esta información es un insumo para establecer estrategias a corto y largo plazo que permitan ganar oportunidades en el mercado de servicios a la empresa, de manera que se tiene en cuenta el grado de vulnerabilidad como información clave para identificar los peligros que se evidencian con la aparición de nuevas empresas con características similares, tecnología avanzada y legalmente autorizadas.

Palabras clave: estructura organizacional; matriz DOFA; análisis de vulnerabilidad; estrategias organizacionales; análisis de mercadeo; alternativas de mejora.

\footnotetext{
${ }^{1}$ Administrador en salud ocupacional de la Corporación Universitaria Minuto de Dios- UNIMINUTO. ORCID: https://orcid.org/0000-0002-9532-4897

${ }^{2}$ Administradora en salud ocupacional de la Corporación Universitaria Minuto de Dios- UNIMINUTO. ORCID: https://orcid.org/0000-0001-9379-2059

${ }^{3}$ Magister en prevención en riesgos laborales, ingeniero industrial, Docente Administración en Seguridad y Salud en el Trabajo, Corporación Universitaria Minuto de Dios- UNIMINUTO. ORCID: https://orcid.org/0000-0002-7711-5004
} 


\title{
Analysis of the organizational structure of \\ Innovadental dental office from the DOFA matrix
}

\begin{abstract}
This work presents the results of the vulnerability quadrant in Innovadental dental office, after performing an analysis of the organizational structure of the office from the DOFA matrix. The quadrants of the vulnerability diagram and the prop line tool were used in order to determine the degree of vulnerability of the organization. This method is a strategic planning tool that was used in this study with the purpose of exploring the environment, and identifying internal and external factors foreseen for planning and determining the improvement actions that help with the growth and strengthening of the company, identifying weaknesses, opportunities, strengths, and threats, as well as recognizing the current state of the organization. This information is an input to establish short and long term strategies in order to gain opportunities in the market of services to the company, so that it takes into account the degree of vulnerability as key information to identify the dangers that are evident with the emergence of new companies with similar characteristics, advanced technology, and that are legally authorized.
\end{abstract}

Keywords: organizational structure; DOFA matrix; vulnerability analysis; organizational strategies; marketing analysis; improvement alternatives.

$* * * * * * * * * * * * * * * * * * * * * * * * * * * * * * * * * * * * * * * * * * * * * * * * * * * * * * * * * * * * * * * * * * * * * * * * * * * * * * * * * * * * * * * * * * * * * * * * * * * * * * * *$
Análise da estrutura organizacional do
Consultorio Innovadental a partir da matriz DOFA

\section{Resumo}

Este trabalho apresenta os resultados do quadrante de vulnerabilidade do consultório odontológico Innovadental, depois de realizar uma análise da estrutura organizacional do consultório a partir da matriz DOFA. Utilizaramse os quadrantes do diagrama de vulnerabilidade e o traço de fatores para determinar o grau no que se encontra a organização. Este método é um instrumento de planejamento estratégico usado no estudo com o fim de explorar o meio, com a identificação de fatores internos e externos previstos para a busca de um planejamento e a determinação de ações de melhora que ajudem ao crescimento e ao fortalecimento da empresa, identificando debilidades, oportunidades, fortalezas e ameaças, bem como reconhecer o estado atual da organização. Esta informação é um insumo para estabelecer estratégias a curto e longo prazo que permitam ganhar oportunidades no mercado de serviços à empresa, de maneira que se tem em conta o grau de vulnerabilidade como informação chave para identificar os perigos que se evidenciam com o surgimento de novas empresas com características similares, tecnologia avançada e legalmente autorizadas.

Palavras-chave: estrutura organizacional; matriz DOFA; análise de vulnerabilidade; estratégias organizacionais; análise de marketing; alternativas de melhora. 
Analyse de la structure organisationnelle de la

clinique Innovadental à partir de la matrice DOFA

\section{Résumé}

Cet article présente les résultats de l'analyse de vulnérabilité du cabinet dentaire Innovadental après réalisation d'une étude - basée sur la matrice SWOT - de la structure organisationnelle de l'entreprise. L'analyse du diagramme de vulnérabilité a été utilisé pour déterminer le degré de fragilité de l'organisation. Cette méthode est un instrument de planification stratégique utilisé pour explorer l'environnement de l'entreprise, identifier les facteurs internes et externes permettant une meilleure planification des actions d'amélioration favorisant croissance et stabilité, identifiant les faiblesses, les opportunités, les forces et les menaces via l'analyse de l'état actuel de l'entreprise. Ces informations servent de données pour établir des stratégies à court et moyen terme permettant de saisir les opportunités du marché des services aux entreprises faisant du degré de vulnérabilité une information clé pour identifier l'imminence des risques provoqués par l'apparition de nouvelles entreprises aux caractéristiques similaires, ou possédant une technologie plus avancée.

Mots-clés: structure organisationnelle; Matrice SWOT; analyse de vulnérabilité; stratégies organisationnelles; analyse marketing; alternatives d'amélioration.

\section{Introducción}

La matriz o análisis DOFA es una herramienta de planeación estratégica de las compañías. Se utiliza con el fin de realizar una evaluación de los factores internos y externos de las organizaciones que, a través de la recolección de información, permite conocer y evaluar las debilidades, las oportunidades, las fortalezas y las amenazas que se identifican en el interior de una empresa, a fin de que implementen, a su vez, estrategias que conlleven a las acciones de mejoras, a aprovechar las fortalezas y trabajar las debilidades encontradas. Esto con el propósito de minimizar o corregir dichas falencias, de manera que se evite la aparición de las amenazas (Ruíz, 2012).

El análisis o matriz DOFA se aplica como estrategia en el consultorio odontológico Innovadental, ubicado en el municipio de Guacarí, Valle, con el objetivo de realizar un análisis de la estructura organizacional del consultorio, a partir de la matriz DOFA, que permita recolectar información para obtener unos resultados del nivel de vulnerabilidad y así tomar acciones correctivas. Esto le permitirá al empresario la prevención de los efectos de estas vulnerabilidades y, a su vez, darlas a conocer al equipo de trabajo, a fin de que se logre una agrupación de estos elementos, trabajar en su mejora y contribuir así al fortalecimiento de la estructura organizacional. 


\section{Metodología}

El análisis DOFA —o FODA, como también se conoce- es un instrumento de planificación estratégica qué se utiliza para la exploración del entorno en la medida en que ayuda a identificar factores tanto internos como externos que deben ser previstos en la búsqueda de una planificación que determine a dónde la empresa debe ir a futuro, según Idalberto Chiavenato (2011). Por el contrario, Ponce-Talancón (2007) afirma que es una alternativa para realizar diagnósticos y determinar estrategias de intervención en las organizaciones o empresas, bien sean productivas o bien sociales. También en este proceso se busca obtener información que permita tomar decisiones correctas y efectivas, de acuerdo con lo que persigue una empresa a futuro, mientras se generan resultados inmediatos. De esta manera, según Carvajal, Paulo, Vásquez y Quishpe (2016), el análisis DOFA tiene amplitud en sus alcances al hacer énfasis en diversos aspectos, a diferencia de otras matrices —p. ej., la matriz de portafolio de negocios, negocios General Electric, Hofer y Schendelque han sido utilizadas por diseñadores de estrategias. Esto en razón a que se considera una estructura conceptual sistemática que facilita la comparación de oportunidades y amenazas externas con las fortalezas y las debilidades de una organización que, utilizada de manera correcta, prevé una base para la formulación de estrategias (Mariño, Cortés y Garzón, 2008).

Es por esto que para la formulación de las estrategias que contribuyan al mejoramiento de la estructura organizacional del consultorio Innovadental se aplica la matriz DOFA —o análisis de vulnerabilidad-, con base en los planteamientos de Humberto Serna (2014), quien aduce que el análisis DOFA «está diseñado para ayudar al estratega a encontrar el mejor acoplamiento entre las tendencias del medio, las oportunidades y amenazas y las capacidades internas, fortalezas y debilidades de la empresa» (p. 188). Esto permitirá al empresario plantear estrategias que le ayuden a minimizar las amenazas utilizando las fortalezas como una fuente de aprovechamiento ante el efecto que pueda causar las debilidades encontradas y así estar en capacidad de prevenir o anticiparse a las amenazas y definir el comportamiento de la empresa a futuro. 
En todo este proceso resulta importante tratar de la planeación estratégica, ya que, según Sánchez (2003), «la planeación estratégica ha cumplido igualmente un papel importante en la formulación de políticas, en la definición de metas e indicadores» (p. 4) que, junto con la aplicación de la matriz DOFA, son esenciales para que la alta gerencia tome decisiones que le permita dar cumplimiento a los objetivos propuestos y alcanzar las metas trazadas.

Dado que este es un modelo diseñado para que los empresarios o estrategas logren elaborar la matriz que les permita identificar las debilidades, las oportunidades, las fortalezas y las amenazas que se encuentran en el interior de la organización, en el medio que la rodea y frente a la competencia, nace la necesidad de implementarla en el consultorio Innovadental, en busca de la identificación de esas falencias y, de esta manera, lograr la toma de decisiones y la implementación de acciones de mejora que propicien el crecimiento empresarial, la permanencia en el mercado y la prestación de servicios de calidad, así como suplir las necesidades de los clientes.

De esta manera, cada vez toma más importancia un exhaustivo diagnóstico en las empresas, ya que de este depende que se logre identificar los problemas que afectan las organizaciones y, por ende, servirá como insumo para la toma de decisiones que permitan el crecimiento y el desarrollo de las empresas con base en sus potenciales.

De acuerdo con lo anterior, Luna (2014, p. 17) señala qué es el diagnóstico y cómo funciona:

Se entiende como diagnóstico: el proceso de investigar cómo funciona la empresa en el presente y obtener información precisa para planear las intervenciones del cambio. El diagnóstico de la empresa también se entiende como la conclusión del estudio de investigación de la situación real de la compañía, que detectan alteraciones negativas, como las debilidades y amenazas, que serán la base para planear el cambio y perfeccionar el funcionamiento.

Frente a la globalización y las exigencias del mercado, la competencia y demás factores tanto internos como externos las empresas se ven en la necesidad de permanecer en un ciclo de mejora que les proporcione una ventaja competitiva. 
En razón a lo anterior, Palacios (2010, p. 18) explica por qué se presentan dichos cambios:

Las compañías de hoy en día se enfrentan, más que nunca, al reto de asimilar fuertes y continuos cambios, no solo de su entorno como: competidores, clientes, proveedores, normas, etc., sino también, del externo en: factores sociales, tecnológicos, económicos, políticos, legales, ambientales y administrativos.

Para cumplir el objetivo se plantea una investigación descriptiva y explicativa con el fin de buscar las causas que originan las debilidades y las amenazas del objeto de estudio. Así, entonces, se establecen los hechos o situaciones que los originan mediante un análisis exhaustivo que permita al empresario tomar medidas de prevención y mejoras en su propósito de crecimiento y fortalecimiento.

\section{Desarrollo}

La matriz o análisis DOFA se aplicó dentro de las instalaciones del consultorio odontológico. Es este un sitio reconocido por médicos para la realización de exámenes, diagnósticos, pronósticos con criterios de prevención y el tratamiento de enfermedades, malformaciones y traumatismos, así como la secuela de estos a nivel de los dientes maxilar y demás tejidos que constituyen el sistema estomatognático (Minsalud, 2013). Innovadental se encuentra ubicada en la calle 7 n. ${ }^{0}$ 7-74, esquina, en el municipio de Guacarí, Valle del Cauca. Presta los servicios de ortodoncia, cirugía, endodoncia, etc., a los habitantes del municipio hace 10 años. Durante la realización de la matriz DOFA se logra establecer con el empresario cuáles son aquellos factores internos y externos de la organización, con el fin de identificar las oportunidades y las amenazas, así como las fortalezas y las debilidades de esta organización.

Durante la recolección y la aplicación de la matriz se aprecia que el consultorio posee espacios acordes para la realización de las actividades y la prestación de los servicios, así como las condiciones higiénicas. Cuenta con una secretaria, dos profesionales - uno fijo y otro por prestación de servicios-y una persona más como personal de aseo. En el comienzo del diagnóstico se encuentran aspectos qué son explícitamente de escasa comunicación, debilidad que puede causar un desbalance en el buen funcionamiento de la entidad. Así 
mismo, se logra identificar que se requiere reorganizar los roles de los trabajadores en el área de canales de comunicación, buscar estrategias que ayuden a los usuarios a identificar los servicios que se prestan, así como la facilidad de encontrar solución a sus necesidades y el buen servicio prestado que ofrece el consultorio.

La presentación de este análisis en cada uno de sus procesos puede ser tanto económico como político, social o cultural, de manera que representa las influencias del ámbito interno de una organización que inciden sobre qué hacer. Esto debido a que pueden favorecer o poner en riesgo el cumplimiento de las metas de la institución o empresa, prever oportunidades y amenazas y brindar la posibilidad de construcción de escenarios anticipados, lo cual posibilita reorientar su rumbo en la parte externa (Instituto Politécnico Nacional , 2002). En el caso de las fortalezas y las debilidades, son aquellos aspectos internos de la empresa que se agrupan bajo esos conceptos, de modo que se realiza una relación entre el estado actual y el futuro necesario para el sostenimiento de la competitividad en general; estos aspectos internos analizados son los que determinan cómo se está frente a la competencia.

Ahora bien, una alternativa rápida en el ámbito de fortalezas y debilidades de la organización corresponde a determinar un listado de estas en el ambiente, lo cual da un ejemplo de una pyme en formación (Lazzari y Maesschalck, 2016).

De acuerdo con este análisis, surgen inquietudes que reflejan la existencia de deficiencias en la aplicación de la herramienta. Diferentes grupos gerenciales y profesionales explican algunos de los defectos en la utilización de la herramienta, argumentando que son causas principales de su aplicación inapropiada. Así, por ejemplo, la identificación de oportunidades como factores que realmente son fortalezas o amenazas en otras que son debilidades, la carencia de claridad al determinar factores del entorno, ubicarse en una parte aislada en el área de influencia del grupo de estudio de la empresa, o dejar de lado los factores internos que sí están bajo su influencia y sobre los cuales se puede actuar. 
Una sugerencia para la generación de ideas y la recopilación de información en el propósito de realizar un buen análisis estratégico es esclarecer los ámbitos que abarcan puntos estratégicos, a fin de identificar el estado actual de la organización (Codina, 2011). Realizar un diagnóstico directo de la estructura y la operación de una organización, en el cual se incluyan los recursos disponibles, las áreas funcionales y las actividades, etc. Tener en cuenta las variables de influencia global como, por ejemplo, la economía, la política, la cultura, la tecnología, las políticas públicas y otras por mencionar fuera de la organización, es importante a fin de determinar tanto oportunidades como fortalezas, puesto que sería un camino ideal en la búsqueda de una correcta aplicación de la herramienta (Rodríguez, Casas, Contreras, Camacho y Andrade, 2015).

Para lograr que una organización, a través de su empresario, esté en capacidad de desarrollar toda su capacidad y habilidad, a fin de aprovechar sus fortalezas y neutralizar o minimizar sus amenazas, es necesario entender y conocer muy bien el significado de cada uno de los ítems, así como conocer que una fortaleza está constituida por aspectos o elementos en los que la venta de sus productos o servicios muestra una superioridad (Ero Del, Vidigal y Adeilson, 2014).

Las fortalezas y las oportunidades son ventajas competitivas. Estas aparecen cuando se adquieren, fundamentalmente, con el esfuerzo personal, de modo que logran crecer las fuertes o aprovecharlas mejor de acuerdo con los demás competidores. Es importante conocerlas porque difícilmente dos empresas pueden presentar iguales ventajas competitivas ante el mercado, debido a que se basan en las variables del entorno.

Las amenazas son variables externas que, si bien afectan a todos los competidores y deben ser potenciadas en diferentes medidas, representan debilidades que toda organización o empresa posee. Las oportunidades y amenazas son aquellas que en definitiva harán posible o no el proyecto, es decir, son aquellas condiciones o situaciones en las que no se tiene ninguna posibilidad de influir (Otero y Gache, 2006). Dentro de este proceso, es necesario mencionar la importancia que tiene, según lo planteado por Acosta y Terán (2015 p.57), «la determinación de las capacidades distintivas para generar ventajas competitivas 
es uno de los objetivos básicos del diagnóstico interno», lo cual resulta ser un factor clave que logran tener las empresas u organizaciones para al alcanzar el éxito frente a la demanda y la competencia.

En el control de gestión de la aplicación del análisis de vulnerabilidad se utiliza una etiqueta lingüística con el fin de lograr la evaluación del estado de las variables, lo que permite medir y comparar las brechas al comprobar los efectos de la aplicación del análisis en cierto momento y considerar así un proceso dinámico (Lazzari y Maesschalck, 2002). Es importante tener en cuenta un control interno que comprenda la estructura, los procedimientos, las políticas y las cualidades del personal de la organización con el objetivo de proteger los activos, promover y fortalecer la eficiencia en las operaciones, asegurar una información válida y, finalmente, estimular y tener una certeza del cumplimiento de las políticas directrices de la empresa (Moreno, 2015).

El análisis del estado de la organización tiene como objetivo identificar múltiples factores que pueden afectar una organización, de manera que se desarrollan un mecanismo - matriz - capaz de expresar la variabilidad conjunta de todas las variables con indicadores claros que permiten efectuar planes de acción y mejoramiento para el cumplimiento de cada uno de los objetivos trazados por la entidad (Cadena, 2015). De igual forma, realizar el diagnóstico y la evaluación del proceso mediante un equipo de trabajo eficiente y determinar así los requerimientos del cliente que dan pie al análisis de las alternativas de mejora.

También, al fortalecer la parte central de esta etapa - que es cumplir con los requerimientos del cliente-, la aplicación del método con exactitud permite de una manera ordenada llevar a cabo la incorporación, la cuantificación y la priorización de las necesidades de la población estudiada, de modo que se disminuyen y eliminan costos innecesarios, se eleva la probabilidad de ventas y se logra que la organización sea más competitiva (Núñez, 2004). 
La elaboración del análisis DOFA es un cuadro de trabajo que permite identificar los factores internos, las fortalezas y las debilidades de la organización, así como factores externos tales como las oportunidades y las amenazas, con el propósito de que se logren plantear planes de acción que permitan trabajar en dichos factores.

En el desarrollo del análisis se deben seleccionar las fortalezas, las oportunidades, las amenazas y las debilidades de mayor impacto en la organización. Así mismo, aquellos factores que puedan afectar el cumplimiento de la misión y la visión de la organización, así como factores económicos, políticos, sociales y culturales que podrían favorecer o poner en riesgo el cumplimiento de los objetivos, en este caso fortalezas y debilidades, factores tanto internos - oportunidades o amenazas - como externos; una vez identificados aquellos elementos se procede a confeccionar la matriz de impacto DOFA (Benjamín, 2011).

Tabla 1. Análisis DOFA

\begin{tabular}{|c|c|}
\hline Fortalezas & Oportunidades \\
\hline $\begin{array}{l}\text { - Innova Dental es reconocida en el territorio municipal } \\
\text { por desarrollar el área quirúrgica a nivel oral de forma } \\
\text { eficaz y eficiente. } \\
\text { - Innova Dental es fuerte en el mercado por tarifas y } \\
\text { nivel de preparación profesional en el área quirúrgica, } \\
\text { oral y de ortodoncia. } \\
\text { - Personal comprometido con el desarrollo y el futuro } \\
\text { de la empresa, dado que se evidencia el sentido de } \\
\text { pertenencia. } \\
\text { - Se busca el mejor diagnóstico para empezar un } \\
\text { tratamiento: } \\
\text {-ortodoncia } \\
\text {-operatoria - calzas de alta estética- } \\
\text {-buenos equipos de trabajo } \\
\text {-tecnología } \\
\text {-equipos de comunicación } \\
\text {-organización en las instalaciones } \\
\text {-buena variedad de servicios } \\
\text {-reconocimiento personal como atracción al cliente }\end{array}$ & $\begin{array}{l}\text { - Buscar que la sociedad conozca y reciba nuestros } \\
\text { servicios a través de canales de comunicación y } \\
\text { capacitación. } \\
\text { - Jornadas de salud a la población. } \\
\text { - Rápida atención en comparación con la competencia. } \\
\text { - Obsequios por tratamientos o servicios. } \\
\text { - Buscar estrategias para que los clientes paguen los } \\
\text { procedimientos; por ejemplo, cuotas. } \\
\text { - Innova opera en la actualidad en un municipio que a } \\
\text { la fecha ha demostrado gran crecimiento poblacional y } \\
\text { económico. } \\
\text { - Nuestros usuarios reflejan una gran connotación con } \\
\text { un buen voz a voz, ya que no refieren dolor } \\
\text { posquirúrgico y sí la habilidad con que son ejecutados } \\
\text { los procedimientos. } \\
\text { - Existen grandes avances que nuestra empresa puede } \\
\text { aprovechar, como, por ejemplo, la tecnología de } \\
\text { cámaras y diseños. }\end{array}$ \\
\hline Debilidades & Amenazas \\
\hline $\begin{array}{l}\text { - Facturar antes del diagnóstico. } \\
\text { - Atención a los clientes en horarios establecidos. } \\
\text { - Comunicación directa con el cliente para brindar el } \\
\text { servicio. }\end{array}$ & $\begin{array}{l}\text { - La falta de comunicación frente al rendimiento de la } \\
\text { organización. }\end{array}$ \\
\hline
\end{tabular}


- Perder clientes por no dar lo que exigen.

- Falta de comunicación, no concuerdan en las decisiones médico y asistente.

- No hay médico de planta para atender urgencias.

- Aquello que no logramos hacer bien e incluso peor que otros se refiere al tema protésico para la rehabilitación total de nuestros pacientes adultos.

- Consideran que una razón de ese problema es la falta de conocimiento y habilidad, y un poco el tema publicitario.

- Viene de la mala asignación de los recursos, ya que se destinan todos en un gran porcentaje al tema de cirugía y ortodoncia.
- Creemos que, con respecto al tema salarial y a las obligaciones de nómina, son mejores los empleados remunerados en otras que en la nuestra.

- Uno de los obstáculos legales es el tema de habilitación de servicios ante la Secretaría de Salud departamental que cada día aumenta su complejidad.

- Claro, la preocupación nuestra es que crece el informalismo de la odontología y la mala competencia de tarifas.

Fuente. Elaboración propia.

La matriz de impacto DOFA, o también llamada ponderada, permitirá relacionar en cada uno de los cuadrantes aquellas fortalezas, oportunidades, debilidades y amenazas que se puedan presentar. Se ubican de forma descendente, lo cual significa que se empezara con las que tengan mayor impacto en cada uno de los cuadrantes (véase la Tabla 2).

Tabla 2. Matriz de impacto DOFA-ponderada

\begin{tabular}{|c|c|c|c|c|c|c|c|}
\hline \multirow[t]{2}{*}{ Fortalezas } & \multicolumn{3}{|c|}{ Impacto } & \multirow[t]{2}{*}{ Oportunidades } & \multicolumn{3}{|c|}{ Impacto } \\
\hline & Alto & Medio & Bajo & & Alto & Medio & Bajo \\
\hline Ortodoncia & $\mathrm{X}$ & & & $\begin{array}{l}\text { Innova opera en un municipio } \\
\text { que a la fecha ha demostrado } \\
\text { alto crecimiento poblacional } \\
\text { y económico. }\end{array}$ & $\mathrm{X}$ & & \\
\hline $\begin{array}{l}\text { Operatoria - calzas de } \\
\text { alta estética- }\end{array}$ & & $\mathrm{X}$ & & $\begin{array}{l}\text { Buscar que la sociedad } \\
\text { conozca y reciba nuestros } \\
\text { servicios a través de canales } \\
\text { de comunicación y } \\
\text { capacitación. }\end{array}$ & $\mathrm{X}$ & & \\
\hline \multirow[t]{2}{*}{ Debilidades } & \multicolumn{3}{|c|}{ Impacto } & Amenazas & \multicolumn{3}{|c|}{ Impacto } \\
\hline & Alto & Medio & Bajo & & Alto & Medio & Bajo \\
\hline $\begin{array}{l}\text { Falta de comunicación; } \\
\text { no concuerdan en las } \\
\text { decisiones médico y } \\
\text { asistente. }\end{array}$ & $\mathrm{X}$ & & & $\begin{array}{l}\text { La falta de comunicación en la } \\
\text { organización para el } \\
\text { rendimiento de esta. }\end{array}$ & $\mathrm{X}$ & & \\
\hline
\end{tabular}




\begin{tabular}{|l|l|l|l|l|l|l|}
\hline $\begin{array}{l}\text { Comunicación directa } \\
\text { con el cliente para } \\
\text { ofrecer los servicios. }\end{array}$ & X & & $\begin{array}{l}\text { Obstáculos legales: la } \\
\text { habilitación de servicios ante } \\
\text { la Secretaría de Salud } \\
\text { Departamental cada día } \\
\text { aumenta su complejidad. }\end{array}$ & $\mathrm{X}$ & \\
\hline
\end{tabular}

Fuente. Elaboración propia.

En el análisis DOFA el cuadro permite que se logren enunciar los factores que son claves para el éxito de las organizaciones. En él se tienen en cuenta los de mayor impacto, de manera que la información consignada en cada cuadrante sirve como insumo para establecer estrategias que lleven al aprovechamiento de las fortalezas y evitar o minimizar así los riesgos o pérdidas económicas u organizativas de la empresa (véase la Tabla 3).

Tabla 3. Análisis DOFA

\begin{tabular}{|c|c|c|}
\hline & Oportunidades & Amenazas \\
\hline & $\begin{array}{l}1 . \\
\text { Innova opera en un municipio que a la fecha } \\
\text { ha demostrado gran crecimiento } \\
\text { poblacional y económico. } \\
2 . \\
\text { Buscar que la sociedad conozca y reciba } \\
\text { nuestros servicios a través de canales de } \\
\text { comunicación y capacitación. }\end{array}$ & $\begin{array}{l}\text { 1. } \\
\text { A falta de comunicación en la organización } \\
\text { para el rendimiento de esta. } \\
2 . \\
\text { Obstáculos legales: la habilitación de } \\
\text { servicios ante la Secretaría de Salud } \\
\text { departamental, pues cada día aumenta la } \\
\text { complejidad para la gestión de esa } \\
\text { habilitación. }\end{array}$ \\
\hline Fortalezas & Estrategias F0 & Estrategias FA \\
\hline $\begin{array}{l}\text { 1. } \\
\text { Ortodoncia } \\
2 . \\
\text { Operatoria -calzas } \\
\text { de alta estética- }\end{array}$ & $\begin{array}{l}\text { 1. } \\
\text { Presentar el portafolio de servicios a los } \\
\text { nuevos habitantes del municipio, de manera } \\
\text { que se reflejen los precios y los medios de } \\
\text { pago. } \\
2 . \\
\text { A través de redes sociales -Facebook, } \\
\text { Instagram, WhatsApp empresarial, correo } \\
\text { electrónico, página web- dar a conocer } \\
\text { servicios, promociones, descuentos, nuevas } \\
\text { tendencias, etc. }\end{array}$ & $\begin{array}{l}\text { Mejorar la comunicación del personal } \\
\text { administrativo de la organización, lo cual } \\
\text { evite efectos negativos tanto económicos } \\
\text { como organizacionales y permita el } \\
\text { aprovechamiento de las fortalezas y así } \\
\text { prevenir o minimizar las debilidades. } \\
2 . \\
\text { Fortalecer el cumplimiento de la normativa } \\
\text { legal ante la Secretaría de Salud y la SST, a fin } \\
\text { de conseguir el aval para la prestación de } \\
\text { nuevos servicios. }\end{array}$ \\
\hline
\end{tabular}




\begin{tabular}{|l|l|l|}
\hline Debilidades & Estrategias Do & Estrategias DA \\
\hline $\begin{array}{l}\text { comunicación: no } \\
\text { concuerdan en las } \\
\text { decisiones médico y } \\
\text { asistente. }\end{array}$ & $\begin{array}{l}\text { Tener una comunicación acertada que } \\
\text { permita asesorar y brindar un servicio } \\
\text { eficiente a sus clientes acorde a sus } \\
\text { necesidades. }\end{array}$ & $\begin{array}{l}\text { Organizar un plan de trabajo en el que se } \\
\text { especifiquen las actividades a realizar, las } \\
\text { responsabilidades y el personal a cargo de } \\
\text { cada una de ellas, logrando el alcance y las } \\
\text { metas trazadas. }\end{array}$ \\
$\begin{array}{l}\text { directa con el cliente } \\
\text { para ofrecer los } \\
\text { servicios. }\end{array}$ & $\begin{array}{l}\text { Tener canales de comunicación directa } \\
\text { asesor-cliente, lo que permita la asignación } \\
\text { de citas y tratamientos, de manera que } \\
\text { ayude a lograr las metas establecidas por la } \\
\text { organización. }\end{array}$ \\
\hline
\end{tabular}

Fuente. Elaboración propia.

El diagrama de vulnerabilidad es un cuadro en el que se realiza la estimación y evaluación de las probabilidades, de manera que se logra identificar qué puede llegar a pasar y el impacto que ocasionaría una amenaza, así como conocer la capacidad de reacción que tiene la empresa ante esta (véase la Tabla 4).

Tabla 4. Diagrama de vulnerabilidad

\begin{tabular}{|c|c|c|c|c|c|c|}
\hline Puntual & Amenaza & Consecuencia & $\begin{array}{l}\text { Impacto } \\
0-10\end{array}$ & $\begin{array}{l}\text { Probabilidad } \\
0-1\end{array}$ & $\begin{array}{l}\text { Reacción } \\
0-10\end{array}$ & $\begin{array}{l}\text { Grado de } \\
\text { vulnerabilidad }\end{array}$ \\
\hline $\begin{array}{l}\text { Innova Dental } \\
\text { es fuerte en el } \\
\text { mercado por } \\
\text { tarifas y en el } \\
\text { nivel de } \\
\text { preparación } \\
\text { profesional del } \\
\text { área quirúrgica } \\
\text { oral y de } \\
\text { ortodoncia. }\end{array}$ & $\begin{array}{l}\text { Llegada de } \\
\text { nuevas } \\
\text { entidades } \\
\text { que ofrezcan } \\
\text { los mismos } \\
\text { servicios a } \\
\text { mejor precio. }\end{array}$ & $\begin{array}{lr}\text { Pérdida } & \text { de } \\
\text { clientes } & y \\
\text { estabilidad } & \\
\text { económica. } & \end{array}$ & 9 & 0,5 & 8 & II \\
\hline $\begin{array}{l}\text { Buscar el mejor } \\
\text { diagnóstico } \\
\text { para empezar } \\
\text { un tratamiento. }\end{array}$ & $\begin{array}{l}\text { Incurrir en } \\
\text { errores de } \\
\text { diagnóstico } \\
\text { para } \\
\text { determinar } \\
\text { el tipo de } \\
\text { tratamiento. }\end{array}$ & $\begin{array}{l}\text { Pérdida } \\
\text { credibilidad por } \\
\text { parte de los } \\
\text { clientes e incurrir } \\
\text { en procesos } \\
\text { legales. }\end{array}$ & 8 & 0,3 & 7 & II \\
\hline $\begin{array}{l}\text { Buenos equipos } \\
\text { de trabajo. }\end{array}$ & $\begin{array}{l}\text { Incremento } \\
\text { de costos por } \\
\text { la } \\
\text { adquisición } \\
\text { de nuevas } \\
\text { tecnologías. }\end{array}$ & $\begin{array}{lr}\text { Pérdida } & \text { de } \\
\text { realización } & \text { de } \\
\text { tratamientos } & \text { y } \\
\text { cirugías } & \text { de } \\
\text { calidad. } & \end{array}$ & 7 & 0,9 & 9 & II \\
\hline
\end{tabular}




\begin{tabular}{|l|l|l|l|l|l|l|}
\hline $\begin{array}{l}\text { Cumplimiento } \\
\text { de las } \\
\text { exigencias } \\
\text { legales. }\end{array}$ & $\begin{array}{l}\text { Inhabilidad } \\
\text { para } \\
\text { prestación de } \\
\text { servicios. }\end{array}$ & $\begin{array}{l}\text { Cierre } \\
\text { consultorio o IPS. }\end{array}$ & & 9 & 0,9 & I \\
\hline
\end{tabular}

Fuente. Elaboración propia.

Los cuadrantes de vulnerabilidad ubican los puntales del diagrama de vulnerabilidad de la empresa para así conocer el grado de vulnerabilidad de una organización y, por ende, la toma de decisiones que conlleve a implementar estrategias que permitan el alcance de las metas y los objetivos organizacionales.

Figura 1. Cuadrantes de vulnerabilidad

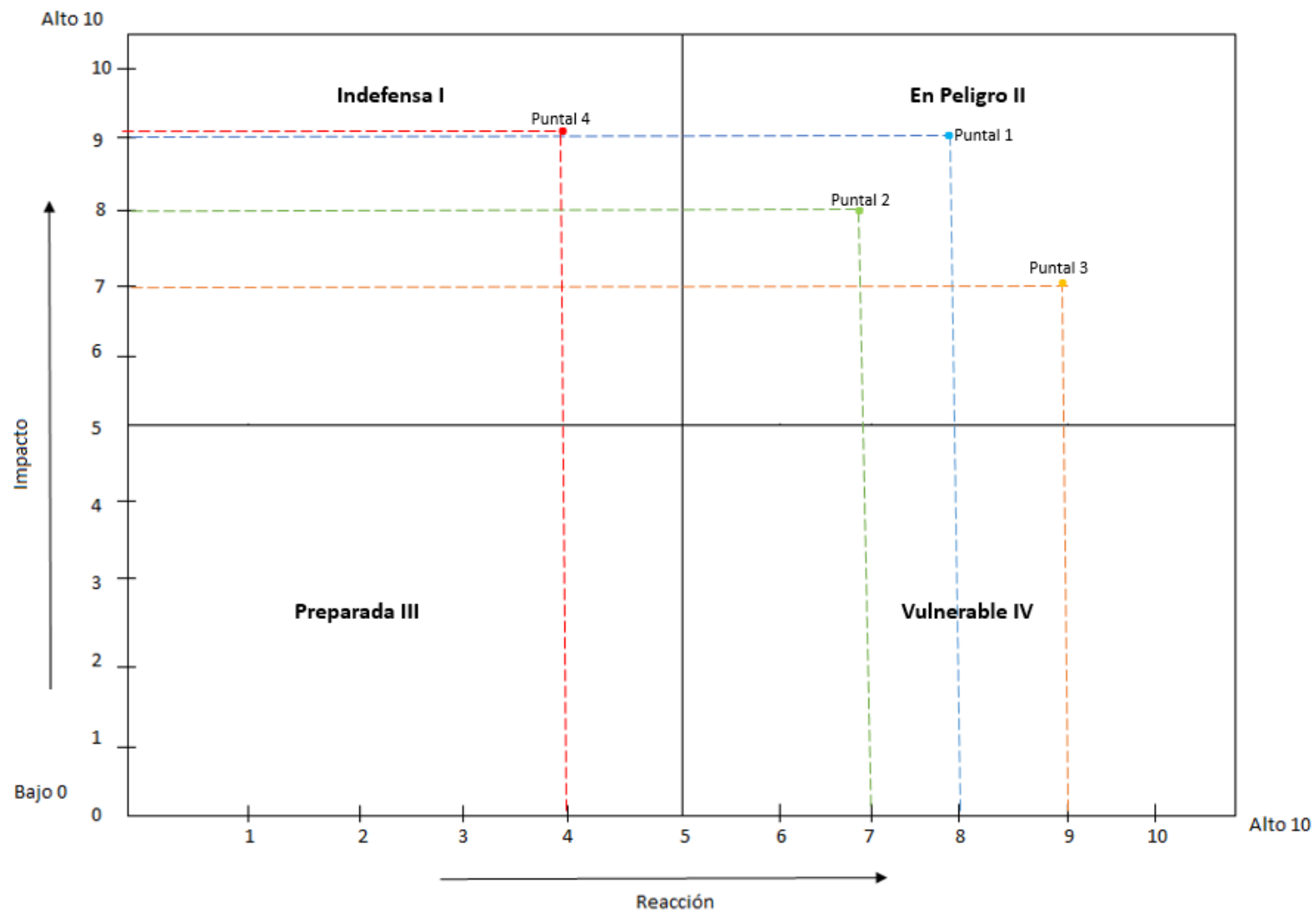

Fuente. Elaboración propia. 


\subsection{Análisis del cuadrante de vulnerabilidad}

El desafío de estudiar procesos emergentes con análisis de ciclo de vida (ACV) ha sido discutido cada vez más (Arvidsson et al., 2018). Por otra parte, se dice que la utilización de aplicaciones fomenta el desarrollo de nuevos modelos de negocio para aumentar la rentabilidad de estos servicios (Gilibert y Ribas, 2019). El análisis del cuadrante de vulnerabilidad permite calificar el estado en que se encuentra la empresa a través de sus respectivos campos del cuadrante.

Serna (2008, p. 197) señala lo siguiente:

La calificación que resulte en el cuadrante I indica que la compañía esta indefensa, en el grado que indique el cuadrante, por tanto, debe tomar acciones inmediatas. El cuadrante II revela que se está en peligro, pero tiene capacidad de reacción, por lo que emprenderá actividades que mejoren su capacidad de reacción. El cuadrante III, muestra que la compañía se encuentra preparada para reaccionar. El cuadrante IV revela amenazas moderadas, frente a las cuales la empresa tiene muy poco que hacer, aunque debe prepararse para reaccionar.

El análisis de vulnerabilidad permite a la organización identificar puntales de los que depende su existencia a la vez que permite clasificar factores que puedan afectar puntales corporativos, también permite determinar el impacto y la capacidad de reacción de la empresa ante sucesos, contribuyendo en la generación de estrategias a corto y largo plazo.

Después de haberse aplicado la matriz DOFA y el análisis de vulnerabilidad se obtienen resultados que permiten conocer el grado de vulnerabilidad de la empresa, de modo que con relación a la figura 1 se obtiene la siguiente información.

Con relación al puntal 1, Innovadental presenta su fuerte en el servicio de ortodoncia, y como amenaza el supuesto de que llegara una empresa que oferte los mismos servicios en Guacarí; ahora bien, la empresa estaría en peligro, pero tendría capacidad de reacción de acuerdo con la calificación del cuadrante I. 
El plan de acción que se le recomienda al empresario es implementar estrategias que le permitan ampliar el portafolio de servicios, hacer uso de las tecnológicas de información y de la comunicación con el propósito de abarcar y establecer un contacto directo con el cliente, de manera que se den a conocer los servicios, las promociones y todo lo relacionado con el consultorio a fin de mantener siempre informados a los usuarios y clientes.

Frente al puntal 2, la oportunidad está en que busca el mejor diagnóstico para empezar un tratamiento. Como amenaza se encuentra que, si llega a presentarse un error en el diagnóstico médico para determinar el tratamiento que requiere el paciente, esto supondría la pérdida de credibilidad por parte de los clientes o problemas legales, de manera que, al analizar el cuadrante, el grado de vulnerabilidad del consultorio es II; así, entonces, con relación al puntal 2 la empresa se encuentra en el cuadrante En Peligro.

El plan de acción depende de la utilización de las herramientas y los equipos adecuados, la asignación de un tiempo determinado que permita identificar el problema con exactitud y la toma de datos relacionados para una intervención precisa.

Con respecto al puntal 3, se cuenta con buenos equipos de trabajo, la empresa invierte su capital en la adquisición de nuevas tecnologías a fin de mejorar la calidad de los servicios y, por tanto, se encuentra en la vanguardia en tecnología; se recomienda capacitar al personal en la utilización adecuada de la herramienta, ya que la organización, de acuerdo con el estudio, se encuentra en el cuadrate En Peligro II.

El plan de acción en este punto se basa en un estudio preliminar de la adquisición de materiales y equipos acorde a las necesidades del consultorio, de manera que le permita realizar inversiones sin pérdida alguna o que no sean innecesarias, tener personal capacitado en el momento de hacer uso de los equipos y proporcionar resultados especiales y eficientes a los usuarios de la organización.

En cuanto al puntal 4, con relación al cumplimiento de los requisitos legales que son exigidos por los entes reguladores, entre ellos la Secretaría de Salud, si el consultorio incumple estos requisitos tiene como amenaza la inhabilidad para la prestación de servicios, 
lo cual supone el cierre del consultorio; en este puntal la empresa presenta un grado de vulnerabilidad de Indefensa I.

Como plan de acción la empresa debe adoptar una estrategia que le permita el cumplimiento de los requisitos legales y, por ende, conseguir el aval para la prestación de nuevos servicios. Se le recomienda que busque el asesoramiento de personal relacionado con este campo, de manera que la oriente para lograr el cumplimiento de las normativas legales vigentes en cuanto a salud, seguridad y salud en el trabajo.

\section{Conclusiones}

El análisis de vulnerabilidad realizado con la información de la matriz DOFA permitió al consultorio odontológico Innovadental comprender las fallas en gestión administrativas que la tienen en los cuadrantes Indefensa y En peligro, evidenciando que está en riesgo la continuidad de la organización ante una posible amenaza generada por la competencia.

Las posibilidades que tiene para mejorar y las herramientas con las que cuenta a fin de sostenerse en el mercado evidencian que son necesarios programas de formación para el personal, con el objetivo de optimizar los tiempos de producción a través del buen manejo de las herramientas tecnológicas con las que se cuenta, así como reducir al máximo, en la medida de lo posible, la probabilidad de sustentar un mal diagnóstico clínico, pues esto afectaría la imagen comercial.

El reconocimiento de su actividad en el mercado y el reconocimiento de sus clientes y usuarios, en cuanto es la esencia principal de su existencia, debe fortalecerse. La implementación de estrategias de mercadeo y sostenibilidad la ayudarán a posicionarse en el mercado, de modo que se deben realizar campañas publicitarias por medio de redes sociales en las que presenten los nuevos servicios y productos para lograr nuevas oportunidades de ingresos. 
Ahora bien, el aprovechamiento de las oportunidades, el fomento de la fortaleza y la prevención de los riesgos identificados en las debilidades y amenazas conllevarán a fundamentar un éxito en el cumplimiento de la misión y la visión, que concluya en el logro de las metas de la organización. Evidenciando así la importancia de la planificación estratégica en las metas de inversión a corto plazo con el objetivo de expandir el nombre del consultorio odontológico.

\section{Referencias}

Acosta, C.; Terán, M. (2015). Administración estratégica. Sangolquí: Universidad de las Fuerzas Armadas ESPE. Recuperado de https://bit.ly/3kYdx90

Arvidsson, R.; Marie-Tillman, A.; Sandén, B.; Janssen, M.; Nordelöf, A.; Kushnir, D.; Molander, S. (2018). Evaluación ambiental de tecnologías emergentes: recomendaciones para un ACV prospectivo. Journal of Industrial Ecology, 22(6), 1286-1294. DOI: https://doi.org/10.1111/jiec.12690

Benjamín, D. P. (2011). El análisis DAFO y los objetivos estratégicos. Contribuciones a la Economía, (3), 1-6.

Cadena, M. V. (2015). Análisis estratégico a partir del análisis de factores. Revista de la Facultad de Ingeniería Industrial, 18(1), 9-28. DOI: https://doi.org/10.15381/idata.v18i1.12062

Carvajal, A.; Paulo, L.; Vásquez, K.; Quishpe, J. (2016). Analisis matriz FODA. Ecuador. Recuperado de https://bit.ly/2Rq0a8N

Chiavenato, I. (2011). Planeación estratégica. fundamentos y aplicaciones. Ciudad de México: McGrawHill Interamericana

Codina, J. A. (2011). Deficiencias en el uso del FODA. Causas y sugerencias. Revista Ciencias Estratégicas, 19(25), 89-100.

Ero l, C. del; Vidigal, F. M.; Adeilson, B. S. (2014). Análisis FODA en el área de validación de una empresa farmacéutica venezolana. Revista de Administração de Roraima-RARR, 4(1), 134-151. DOI: https://doi.org/10.18227/rarr.v4i1.1948

Gilibert, M.; Ribas, I. (2019). Sinergias entre los servicios de movilidad compartida relacionados con el automóvil basados en aplicaciones para el desarrollo de modelos de negocio más rentables. Journal of Industrial Engineering and Management, 12(3). 405-420. DOI: https://doi.org/10.3926/jiem.2930

Instituto Politécnico Nacional. (Marzo de 2002). Metodología para el análisis FODA. Ciudad de México, México.

Lazzari, L. L.; Maesschalck, V. (2002). Control de gestión: una posible aplicación del análisis FODA. Cuadernos del Cimbage, (5), 71-90. 
Lazzari, L.; Maesschalck, V. (2016). El análisis FODA como una herramienta para el control de gestión. Cuadernos del Cimbage, (5), 71-90.

Luna, A. (2014). Administración estratégica. México: Patria.

Mariño, I. A.; Cortés, A. F.; Garzón, R. L. (2008). Herramienta de software para la enseñanza y entrenamiento en la construcción de la matriz DOFA. Revista Ingeniería e Investigación, 28(3), 159-164.

Minsalud (Ministerio de Salud y Protección Social). (2013). Resolución 1441 de 2013 [presentación de diapositivas]. Recuperado de https://bit.ly/3wlumAz

Moreno, V. L. (2015). El control interno como herramienta de gestión corporativa (Trabajo final diplomado). Universidad Militar Nueva Granada, Colombia Recuperado de https://bit.ly/36djn2f

Núñez, L. V. (2004). Aplicación de una metodología de mejora de procesos basada en el enfoque de gestión por procesos, en los modelos de excelencia y el QFD en una empresa del sector de confecciones de Barranquilla (Colombia). Ingeniería \& Desarrollo, (16), 45-58.

Otero, D.; Gache, F. (2006). Evoluciones dinámicas en el diagrama FODA. Revista Científica «Visión de Futuro», 6(2), 1-6.

Palacios, L. (2010). Dirección estratégica. Fundamentos de la dirección estratégica. (2ª ed., 12-18). Bogotá: Ecoe.

Ponce-Talancon, H. (2007). La matriz foda: alternativa de diagnóstico y determinación de estrategias de intervención en diversas organizaciones. Enseñanza e Investigación en Psicología, 12(1), 113-130.

Rodríguez, T. A.; Casas, P. D.; Contreras, L. G.; Camacho, R. J.; Andrade, G. A. (2015). Análisis de un programa médico-asistencial para diabetes (diabetimss) mediante el método FODA. Revista Médica de la Universidad de Costa Rica, 9(1), 55-65. DOI: https://doi.org/10.15517/rmu.v9i1.19645

Ruíz, X. (2012). Análisis DOFA. Universidad Nacional de Colombia. Bogota, Colombia. Recuperado de https://bit.ly/33bqaYE

Sánchez, J. (2003). Estrategia integral para pymes innovadoras. Revista Escuela de Administración de Negocios, (47), 34-45.

Serna, G. H. (2008). Gerencia estratégica (10ª ed.). Bogotá: 3R Editores.

Serna, H. (2014). Gerencia estratégica. En Análisis DOFA análisis de vulnerabilidad. (11ª ed., 187-198). Bogotá: Panamericana 